\title{
A comparison of Bonfils intubation fiberscopy and fiberoptic bronchoscopy in difficult airways assisted with direct laryngoscopy
}

\author{
Soo Hwan $\mathrm{Kim}^{1}$, Su Jin $\mathrm{Woo}^{1}$, and Jong Hoon Kim² \\ ${ }^{1}$ Department of Anesthesiology and Pain Medicine, Kangnam Sacred Heart Hospital, Hallym University College of Medicine, Seoul, \\ ${ }^{2}$ Office of Medical Education, Inha University School of Medicine, Incheon, Korea
}

Background: To evaluate the usefulness of Bonfils intubation fiberscope assisted by direct laryngoscopy (BIFDL) and flexible fiberoptic bronchoscope assisted by direct laryngoscopy (FOB-DL) using video recording in cases of unanticipated difficult intubation with respect to the time required to visualize the vocal cords and place the endotracheal tube. We compared two fiberscopes in patients with authentic difficult airways.

Methods: In this randomized, controlled clinical trial, 40 patients (grade 3 according to grades of difficulty in laryngoscopy), scheduled for surgery under general anesthesia were randomly allocated to BIF-DL group or FOB-DL group. Number of attempts, time required for visualization of the vocal cord (T1) and placement of the endotracheal tube (T2) from insertion of instrument during the last successful attempt, and duration of scope manipulation during all attempts $\left(\mathrm{T}_{\text {total }}\right)$ were recorded. If intubation failed with one method, the other method was tried; these cases were then excluded. The incidence of sore throat and hoarseness was assessed.

Results: T1, T2, and $\mathrm{T}_{\text {total }}$ were significantly shorter in BIF-DL group (T1: $21.9 \pm 8.2 \mathrm{sec}$ vs. $80.4 \pm 29.9 \mathrm{sec}, \mathrm{P}<0.001$, $\mathrm{T}_{\text {total }}: 77.9 \pm 41.2 \mathrm{sec}$ vs. $145.5 \pm 83.9 \mathrm{sec}, \mathrm{P}=0.003$ ). In two cases, it was impossible to intubate with BIF-DL, but the procedure was subsequently successful using fibreoptic bronchoscope.

Conclusions: Intubation of difficult airways can be performed more rapidly with BIF-DL, but sometimes it may not be possible to intubate with the scope. (Korean J Anesthesiol 2010; 58: 249-255)

Key Words: Bronchoscopes, Fiberoptics, Intubation, Laryngoscopy, Video recording.

Received: October 7, 2009. Revised: October 22, 2009. Accepted: October 28, 2009.

Corresponding author: Jong Hoon Kim, M.D., Ph.D., Office of Medical Education, Inha University School of Medicine, 3 ga, Shinheung-dong, Jung-gu, Incheon 400-712, Korea. Tel: 82-32-890-0918, Fax: 82-32-888-7224, E-mail: minasun01@inha.ac.kr

ㄷ) This is an open-access article distributed under the terms of the Creative Commons Attribution Non-Commercial License (http:// creativecommons.org/licenses/by-nc/3.0/), which permits unrestricted non-commercial use, distribution, and reproduction in any medium, provided the original work is properly cited. 


\section{Introduction}

Failed or difficult endotracheal intubation is an important cause of morbidity and mortality during anesthesia [1]. Several methods have been introduced to identify patients who are in danger of difficult intubation before the initiation of anesthesia $[2,3]$. Not all cases can be identified before anesthesia, however, and many cases of difficult intubation arise after trying to find the vocal cords by direct laryngoscopy once unconsciousness has been induced and the skeletal muscles relaxed. The actual difficulties surrounding intubation can only be determined by grading the exposure of the vocal cords by conventional direct laryngoscopy [4]. A number of devices are available to manage the difficult airway including flexible fiberoptic bronchoscopes, the rigid optical stylet, light wands, rigid fiberscopes, the Bullard laryngoscope, the Augustine Scope, the intubation laryngeal mask airway, and a visualized endotracheal tube [5].

Among the preferred methods, flexible fiberoptic bronchoscope (FOB), which used to be regarded as the gold standard in planning predicted difficult airway management $[6,7]$, but numerous hours of training are necessary to optimize control of FOB [8,9]. Unlike FOB, Bonfils intubation fiberscope (BIF) can be easier to control than FOB $[5,10]$, because BIF is covered by hard metal and its end is fixed and slightly curved upward. In addition, BIF requires a short preparation time and it can be used quickly in an emergency situation [11]. Although intubation can be done using an FOB or BIF without any assisting device, even in difficult airways, there have been several studies that the difficult intubation can be facilitated by direct laryngoscopy (DL) [11-13]. Accordingly, it might be preferable to perform intubation using FOB or BIF assisted by direct laryngoscopy in difficult intubation patients.

The main objective of this study was to evaluate the FOB assisted by direct laryngoscopy (FOB-DL) and BIF assisted by direct laryngoscopy (BIF-DL) using video recording in cases of unanticipated difficult intubation with respect to the time required to visualize the vocal cords and place the endotracheal tube in the last successful attempt. In addition, the number of intubation attempts and duration FOB or BIF manipulation were observed.

\section{Materials and Methods}

After protocol approval by the institutional review board (IRB), 40 patients scheduled for elective surgery under general endotracheal anesthesia between January 2006 and December 2007, were selected in this study. We did not seek informed consent since airway management strictly followed the standard operating procedures of our institution which, in turn were based on the ASA difficult airway algorithm [14], but patients gave written consent to data analysis and publication. The order of patients intubated using FOB or BIF was decided randomly by the order of odd (FOB) or even (BIF) numbers from a random number table. Patients with a history of difficult or impossible mask ventilation, morbidly obese (BMI > $35 \mathrm{~kg}$ / $\mathrm{m}^{2}$ ), or known anatomical anomalies of the airway (i.e., limited mouth opening, limited cervical spine mobility, retrognathia, Cruzon syndrome and Pierre Robin sequence), and younger than age 8 (or who required an endotracheal tube smaller than an internal diameter of $6.0 \mathrm{~mm}$ ), were excluded. Patients were also excluded if oxygenation could not be maintained by mask ventilation, or if performing a direct laryngoscopy had the potential to cause harm, such as with cervical spine instabilities.

After pre-oxygenation via a facemask for $3 \mathrm{~min}$, anesthesia was induced with $2 \mathrm{mg} / \mathrm{kg}$ propofol or $5 \mathrm{mg} / \mathrm{kg}$ thiopental sodium. After adequate mask ventilation with $100 \%$ oxygen and 3 vol\% sevoflurane was ensured, $0.6 \mathrm{mg} / \mathrm{kg}$ rocuronium was administered to facilitate tracheal intubation. After adequate neuromuscular blockade was confirmed with double burst stimulation using a nerve stimulator (Innervator $252^{\circledR}$, Fisher \& Paykel Healthcare, Auckland, New Zealand), the patient's head was put in the sniffing position. Direct laryngoscopy was then performed using a Macintosh blade size 3 or 4, as appropriate, and the laryngoscopic view graded according to Cormack \& Lehane [4].

The subjects of this study were patients whose vocal cords were not visualized; only the epiglottises were seen by direct laryngoscopy (grade 3 according to grades of difficulty in laryngoscopy). At least two certified anesthesiologists, who were specialized in airway management, confirmed the grade of difficulty for each patient. The anesthesiologists checked to see if the grade was changeable by head elevation or pressing on the thyroid cartilage. If the grade declined to 2 or 1 as a result of these maneuvers, the patient was excluded from the study.

After the presence of a difficult airway was confirmed, endotracheal intubation was tried with FOB-DL or BIF-DL with a Macintosh blade size 3 or 4, as appropriate, according to the order determined by random numbers. Direct laryngoscope was manipulated by a 3 -yr resident in anesthesiology in FOB-DL group, and by operator by himself in BIF-DL group (Fig. 1, 2). The endotracheal tubes used in this study were conventional (Hi-Lo ${ }^{\mathrm{TM}}$, Mallinckrodt, Athlone, Ireland) or reinforced (SafetyFlex $^{\mathrm{TM}}$, Mallinckrodt, Athlone, Ireland) endotracheal tubes. A flexible fiberoptic bronchoscope with an external diameter of 3.8 mm (PortaView ${ }^{\circledR}$ LF-GP, Olympus optical co., ltd., Tokyo, Japan) was used for the FOB-DL group. A Bonfils intubation fiberscope with an external diameter of $5.0 \mathrm{~mm}$ (Bonfils ${ }^{\circledR}$, Karl Storz Endoscope, Tuttlingen, Germany) was used for the BIFDL group and inserted using a midline approach with the uvula and epiglottis as landmarks. During intubation, the FOB and 


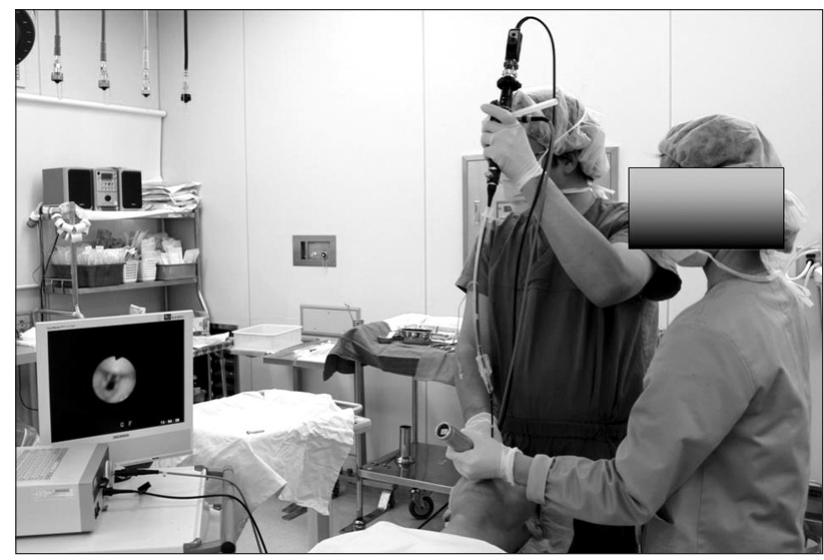

Fig. 1. Intubation with a flexible fiberoptic bronchoscope assisted by direct laryngoscopy. The video camera is attached at the eyepiece of the scope. Note that the assistant holds the direct laryngoscope and the anesthesiologist manipulates the bronchoscope with two hands.

BIF were attached to the camera of a video processing system (Tele Pack, Karl Storz-endoscope, Tuttlingen, Germany). The FOB and BIF were operated by the only two certified anesthesiologists specialized in airway management and familiar with both devices. If intubation was not successful at the first attempt, it was retried with the same device up to a maximum of five times until intubation was successful. One attempt was defined as a single passage of the device until it was withdrawn again. Mask ventilation was maintained between attempts. If it was determined that intubation was impossible with the first device by at least two certified anesthesiologists, the second device was attempted, and the reason for failure was recorded. These cases were excluded from comparisons between groups.

Videos of all endoscopies were converted into video files ( ${ }^{*}$.avi) and stored in a computer connected with Tele Pack. Endoscopy duration was measured by replaying the video files after the procedures were complete. The following parameters were all measured by another anesthesiologist who did not participate in the procedures - number of intubation attempts, the time frame from the point of FOB or BIF insertion until the vocal cords were visualized (T1), the time frame from the point of FOB or BIF insertion until the placement of the endotracheal tube (T2), and the total duration of FOB or BIF manipulation $\left(\mathrm{T}_{\text {total }}\right)$. The parameters $\mathrm{T} 1$ and $\mathrm{T} 2$ are measured only on the last successful attempt, and $\mathrm{T}_{\text {total }}$ was measured from the first attempt until the last successful attempt, and does not include mask ventilation periods during intervals of failed intubation attempts. The measurement of time at unsuccessful attempts began at the moment the endoscope was inserted into the mouth opening and stopped at the moment the endoscope was withdrawn from the patient. $\mathrm{T}_{\text {total }}, \mathrm{T} 1$, and $\mathrm{T} 2$ were measured

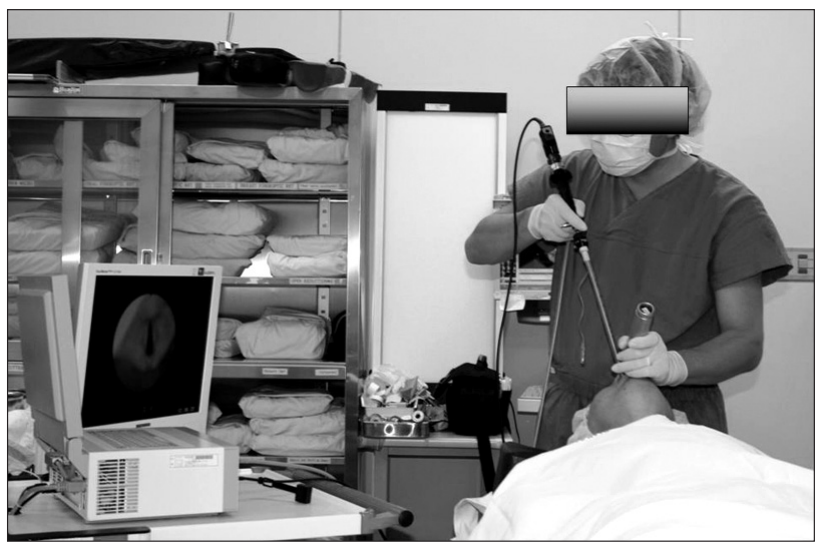

Fig. 2. Intubation with a Bonfils intubation fiberscope assisted by direct laryngoscopy. The video camera is attached at the eyepiece of the scope. Note that the anesthesiologist holds the direct laryngoscope with his left hand and manipulates the fiberscope with his right hand.

by checking the time scale of the video-playing software. The time required to initially prepare the FOB or BIF, for mask ventilation, and for preparation between intubation attempts, was excluded. Mean arterial blood pressure and pulse rate were measured just before the first direct laryngoscopy and just after successful intubation. Sore throat and hoarseness were checked by another anesthesiologist who was blinded to this study using visual analogue scales (VAS, ranges 0 to 10) 24 hours after surgery.

We considered a difference of 20 seconds on the time required to place the endotracheal tube successfully from the beginning of the procedure (T2) to be clinically relevant. A standard deviation of 20 seconds was estimated for this difference. On the basis of the formula for normal theory and assuming a twosided error type I error of 0.05 and a power of $0.90,13$ patients in each group were required to show a difference in the mean time of 20 seconds between two groups. We elected to evaluate 20 patients in each group (i.e., $25 \%$ more) to compensate for possible protocol violations during the study period. Differences between the two groups in endoscopy duration, mean arterial blood pressure, pulse rate, and patient characteristics such as height, weight, and age were compared with an unpaired Student's t-test. The number of intubation attempts between the two groups was compared with a Chi-square test. The incidence and severity of sore throat and hoarseness were compared with the Chi-square test and Mann-Whitney U-test. P values less than 0.05 were considered to indicate a significant statistical difference between the two groups.

\section{Results}

Patient characteristics were not significantly different between 
the two groups (Table 1). Intubation was successful at the first attempt in about half of the cases, and the number of intubation attempts was not significantly different between groups (Table 2). All measures of endoscopy duration (T1, T2 and $\mathrm{T}_{\text {total }}$ ) were significantly shorter in the BIF-DL group (T1: $21.9 \pm 8.2 \mathrm{sec}$ vs. $32.1 \pm$ $14.0 \mathrm{sec}, \mathrm{P}=0.011$, T2: $41.3 \pm 20.3 \mathrm{sec}$ vs. $80.4 \pm 29.9 \mathrm{sec}, \mathrm{P}<0.001$,

Table 1. Patient Characteristics

\begin{tabular}{lcc}
\hline & FOB-DL group & BIF-DL group \\
\hline Age (years) & $48 \pm 21$ & $56 \pm 14$ \\
Sex (M/F) & $14 / 6$ & $13 / 7$ \\
Height (cm) & $162 \pm 17$ & $162 \pm 11$ \\
Weight (kg) & $63 \pm 17$ & $68 \pm 12$ \\
ASA class & & \\
1 & 7 & 9 \\
2 & 9 & 8 \\
3 & 4 & 3 \\
\hline
\end{tabular}

Data are mean \pm SD or number. There were no significant differences between groups. BIF-DL: Bonfils intubation fiberscope by direct laryngoscope, FOB-DL: Fiberoptic bronchoscope by direct laryngoscope.

Table 2. Comparison of Number of Intubation Attempts

\begin{tabular}{ccc}
\hline Attempts & FOB-BL group (n/\%) & BIF-BL group (n/\%) \\
\hline 1 & $10(50)$ & $11(55)$ \\
2 & $2(10)$ & $3(15)$ \\
3 & $2(10)$ & $2(10)$ \\
4 & $2(10)$ & $2(10)$ \\
5 & $4(20)$ & $2(10)$ \\
\hline
\end{tabular}

Data are numbers (\%). No significant differences between groups were observed. BIF-DL: Bonfils intubation fiberscope by direct laryngoscope, FOB-DL: Fiberoptic bronchoscope by direct laryngoscope.

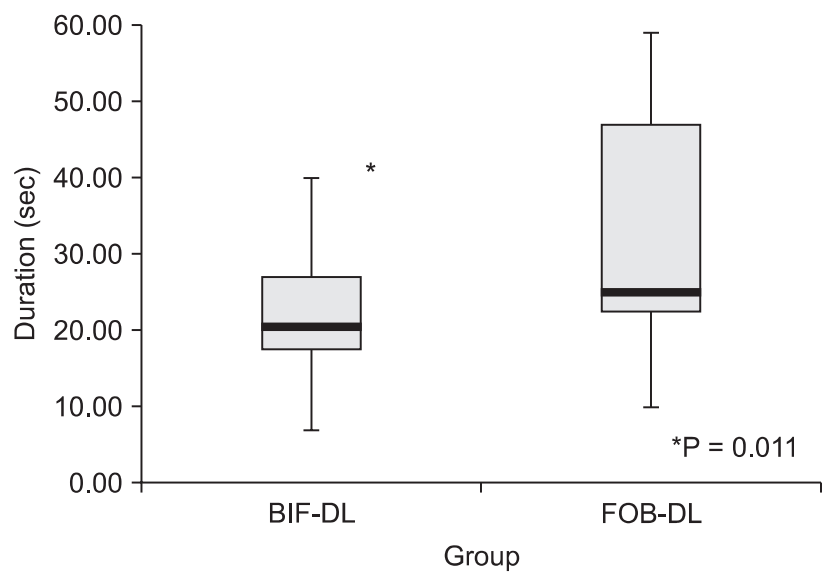

Fig. 3. Comparison of the time required to visualize the vocal cords during the last successful attempt (T1) between the two groups. T1 was significantly shorter in the Bonfils intubation fiberscope (BIF) group. Box plot shows the median (thick line in the box), interquartile range (box), and range (whiskers).
$\mathrm{T}_{\text {total }}: 77.9 \pm 41.2 \mathrm{sec}$ vs. $145.5 \pm 83.9 \mathrm{sec}, \mathrm{P}=0.003$ ) (Fig. $3-5$ ). In two cases, it was impossible to intubate with BIF, but subsequent intubation was achieved with FOB. Pulse rate and mean arterial blood pressure were not significantly different between the two groups both before and after intubation (Table 3).

Three patients from FOB-DL group and one from BIF-DL group complained of sore throat (VAS $<3$ ). Two patients from FOB-DL group and one from BIF-DL group complained of hoarseness (VAS $<3$ ). The scores of sore throat or hoarseness according to the visual analogue scale were not statistically different between groups.

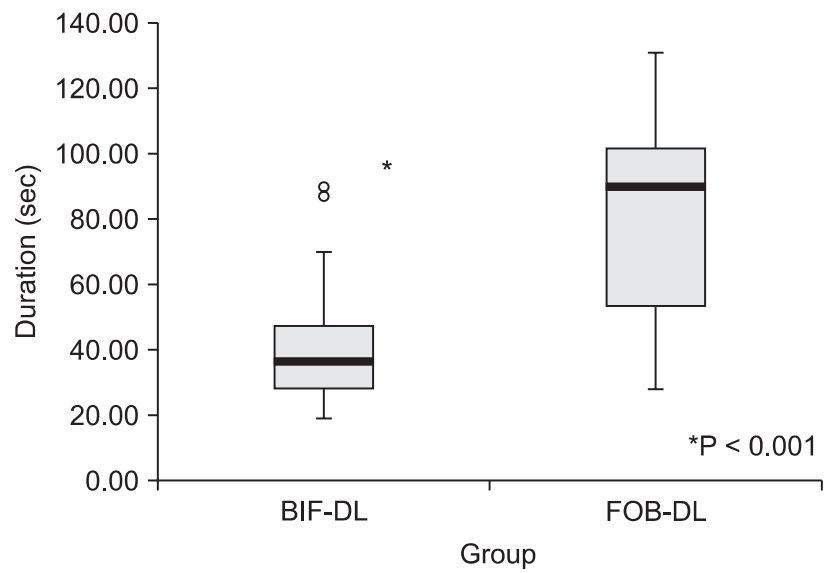

Fig. 4. Comparison of the time required to place the endotracheal tube in the last successful attempt (T2) between the two groups. $\mathrm{T} 2$ was significantly shorter in the Bonfils intubation fiberscope (BIF) group. The box plot shows the median (thick line in the box), interquartile range (box), range (whiskers), and outliers (small circle).

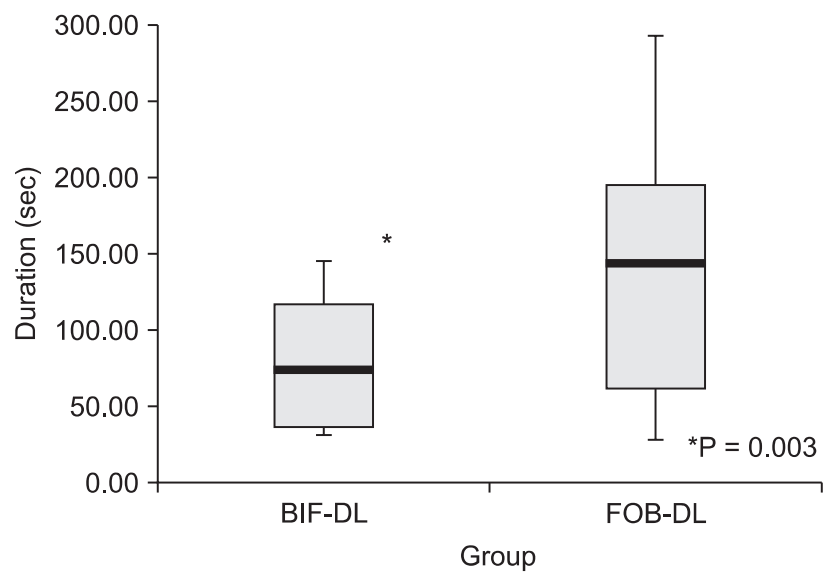

Fig. 5. Comparison of the duration of scope manipulation during the entire attempt $\left(\mathrm{T}_{\text {total }}\right)$ between the two groups. $\mathrm{T}_{\text {total }}$ was significantly shorter in the Bonfils intubation fiberscope (BIF) group. The box plot shows the median (thick line in the box), interquartile range (box), and range (whiskers). 
Table 3. Mean Arterial Blood Pressure and Pulse Rate just before the Intubation Attempt and After Completion of Intubation

\begin{tabular}{lcc}
\hline & FOB-BL group BIF-BL group \\
\hline $\begin{array}{l}\text { Mean arterial blood pressure (mmHg) } \\
\quad \text { Before intubation }\end{array}$ & $75 \pm 8$ & $73 \pm 9$ \\
$\quad$ After intubation & $94 \pm 8$ & $91 \pm 9$ \\
Pulse rate (/min) & & \\
$\quad$ Before intubation & $70 \pm 10$ & $67 \pm 11$ \\
After intubation & $76 \pm 10$ & $75 \pm 12$ \\
\hline
\end{tabular}

Data are mean \pm standard deviation. There were no significant differences between groups. BIF-DL: Bonfils intubation fiberscope by direct laryngoscope, FOB-DL: Fiberoptic bronchoscope by direct laryngoscope.

\section{Discussion}

Many methods and instruments have been developed to assist difficult intubation [15-19]. Most approaches emphasize visualizing vocal cords that cannot easily be seen by direct laryngoscopy [20-24]. In this study, we compared the effectiveness of two tools, the BIF-DL and FOB-DL, in the same situation (grade 3 difficulty of laryngoscopy). Many methods have been suggested to facilitate FOB or BIF use, such as jaw thrust, use of devices, lingual traction, direct laryngoscopy, or a combination of methods [11-13]. Direct laryngoscopy has either proven to be more helpful than other methods in assisting FOB use [12], or at least has been no worse for FOB use in the general population [13]. Hagberg and Westhofen [25] also reported a useful two-person technique using FOB in combination with direct laryngoscopy for extubation/reintubation in two intensive care unit patients with known difficult airways. With direct laryngoscopy, the space available in the oral cavity to manipulate the scopes can be expanded, and the epiglottis can be elevated to secure the pathways of the scopes to the vocal cords. Accordingly, we selected direct laryngoscopy to facilitate orotracheal intubation using either FOB or BIF in difficult airways.

In this study, all evaluated time parameters were significantly shorter in BIF-DL group, demonstrating that intubation was faster with BIF-DL than FOB-DL in difficult airways. We felt it was important to measure not only the entire time needed to complete the intubation, but also the time of the last successful attempt. It should be noted that intubation failure was not always a direct result of the instrument being used. For example, direct laryngoscopy also affected the success of the procedure. Sometimes the tip of the direct laryngoscope was located at the wrong place, or slipped away from the vallecula during the procedure. In such cases, the length of the procedure was affected by operator error rather than actual airway difficulties. By evaluating the time needed for the successful attempt, we ensured that the intubation was performed under optimum conditions with minimal human errors. Nevertheless, all time

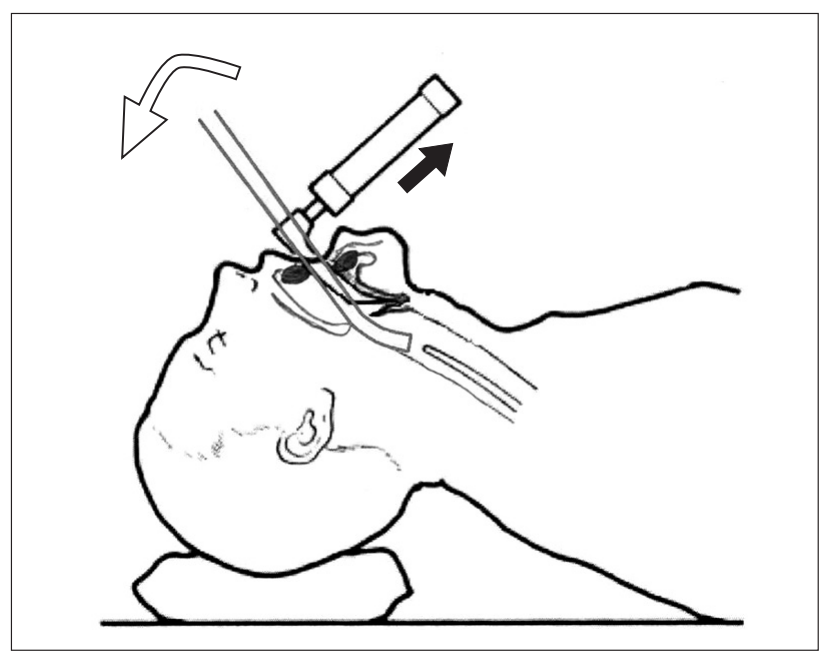

Fig. 6. Illustration of the vertical section of Bonfils intubation fiberscopy. In this case, intubation was impossible because the endotracheal tube was stuck between the teeth and shaft of the scope when the operator tried to visualize the vocal cords (the proximal part must be pushed down towards the direction of arrow to lift up the distal end of the scope). The intubation was subsequently achieved with flexible fiberoptic bronchoscopy.

parameters were shorter in the BIF-DL group, suggesting that it may be easier to intubate difficult airways with BIF regardless of human error. The time parameters were measured accurately, because the whole procedure was recorded by a video processing system with cameras attached to the scope eyepieces. Attaching the camera to the scopes might be another advantage of using BIF. Operators can manipulate the direct laryngoscope and BIF simultaneously, because BIF can be manipulated with one hand if the video camera is present (Fig. 1). FOB must be manipulated by both hands of the operator, making it necessary for an assistant to handle the direct laryngoscope (Fig. 2). Verbal communication between the operator and assistant is needed for optimal intubation, inevitably causing a time delay. In contrast, the BIF operator could optimize intubation without communicating with an assistant. The duty of the assistant in BIF-DL group was to insert the endotracheal tube at the request of the operator, a much simpler communication compared to FOB-DL group. In FOBDL group, the endotracheal tube was inserted by the operator.

Insertion of the endotracheal tube through the vocal cords might also explain the observed time delay in FOB-DL group. FOB was passed through the vocal cords, and then the tube was inserted by using the shaft of FOB as a stylet. Moreover, sometimes the tube could not be inserted the first time after passing the scope in the FOB-DL group, requiring rotation to pass through the vocal cords. In contrast, in the BIF-DL group, the tube could be inserted on first attempt right after visualizing the vocal cords without passing the scope through the vocal 
cords in almost all cases. These technical distinctions could explain why the difference in $\mathrm{T} 2$ was larger than the difference in $\mathrm{T} 1$ between the two groups.

Intubation with the BIF was impossible in two cases. In one case, the vocal cord could not be visualized. In the other case, intubation was impossible due to a failure to advance the endotracheal tube because it was stuck between the teeth and shaft of the BIF (Fig. 6). Intubation was successful in both cases with FOB-DL. All cases in which FOB-DL was tried first were successful, although more time was needed to complete intubation.

Rudolph et al. [26] reported comparable results using similar methods, although there were some differences with our study. They did not use a direct laryngoscope in all cases, which may have made some procedures more difficult. It is also not clear if their time measurements included equipment preparation or ventilation time between attempts. We felt that preparation time should be excluded to focus on the true performance of the two scopes. If intubation was not successful in the first attempt, patients would be ventilated between attempts, and ventilation time would vary case by case. They indicated that there is likely a difference in the duration of procedures between the two scopes in real clinical settings. Because of our study design, our study probably reflects the mechanical differences between the use of the two scopes more accurately. Finally, they did not use a video camera attached to the eyepiece to record the procedures.

In conclusion, orotracheal intubation was performed more rapidly and easily with BIF-DL than FOB-DL in patients with grade 3 difficult airways. Rapid securement of the airway is unquestionably the most important task in patients presenting with difficult airway and laryngoscopy. Despite the $10 \%$ failure rate with BIF-DL, we recommend the use of BIF first over FOB with the assistance of direct laryngoscopy. However, considering that it was not always possible to intubate with BIFDL, FOB should be available as a backup choice.

\section{References}

1. Benumof JL. Management of the difficult adult airway. With special emphasis on awake tracheal intubation. Anesthesiology 1991; 75: 1087-110.

2. Mallampati SR, Gatt SP, Gugino LD, Desai SP, Waraksa B, Freiberger $D$, et al. A clinical sign to predict difficult tracheal intubation: a prospective study. Can Anaesth Soc J 1985; 32: 429-34.

3. Redick LF. The temporomandibular joint and tracheal intubation. Anesth Analg 1987; 66: 675-6.

4. Cormack RS, Lehane J. Difficult tracheal intubation in obstetrics. Anaesthesia 1984; 39: 1105-11.

5. Kitamura T, Yamada Y, Du HL, Hanaoka K. Efficiency of a new fiberoptic stylet scope in tracheal intubation. Anesthesiology 1999;
91: 1628-32

6. Rosenblatt WH, Wagner PJ, Ovassapian A, Kain ZN. Practice patterns in managing the difficult airway by anesthesiologists in the United States. Anesth Analg 1998; 87: 153-7.

7. Bokhari A, Benham SW, Popat MT. Management of unanticipated difficult intubation: a survey of current practice in the Oxford region. Eur J Anaesthesiol 2004; 21: 123-7.

8. Cole AF, Mallon JS, Rolbin SH, Ananthanarayan C. Fiberoptic intubation using anesthetized, paralyzed, apneic patients. Results of a resident training program. Anesthesiology 1996; 84: 1101-6.

9. Ovassapian A, Yelich SJ, Dykes MH, Golman ME. Learning fibreoptic intubation: use of simulators v. traditional teaching. $\mathrm{Br} \mathrm{J}$ Anaesth 1988; 61: 217-20.

10. Halligan M, Charters P. A clinical evaluation of the Bonfils Intubation Fibrescope. Anaesthesia 2003; 58: 1087-91.

11. Bein B, Yan M, Tonner PH, Scholz J, Steinfath M, Dorges V. Tracheal intubation using the Bonfils intubation fibrescope after failed direct laryngoscopy. Anaesthesia 2004; 59: 1207-9.

12. Smith JE, Mackenzie AA, Scott-Knight VC. Comparison of two methods of fibrescope-guided tracheal intubation. Br J Anaesth 1991; 66: 546-50.

13. Stacey MR, Rassam S, Sivasankar R, Hall JE, Latto IP. A comparison of direct laryngoscopy and jaw thrust to aid fibreoptic intubation. Anaesthesia 2005; 60: 445-8.

14. American Society of Anesthesiologists Task Force on Management of the Difficult Airway. Practice guidelines for management of the difficult airway. An updated report. Anesthesiology 2003; 98: 126977.

15. Bjoraker DG. The Bullard intubating laryngoscopes. Anesthesiol Rev 1990; 17: 64-70.

16. Cooper RM. Use of a new videolaryngoscope (GlideScope) in the management of a difficult airway. Can J Anaesth 2003: 50: 611-3.

17. Krafft P, Krenn CG, Fitzgerald RD, Pernerstorfer T, Fridrich P, Weinstabl C. Clinical trial of a new device for fiberoptic orotracheal intubation (Augustine Scope). Anesth Analg 1997; 84: 606-10.

18. Pearce AC, Shaw S, Macklin S. Evaluation of the Upsherscope. A new rigid fibrescope. Anaesthesia 1996; 51: 561-4.

19. Weiss M. Video-intuboscopy: a new aid to routine and difficult tracheal intubation. Br J Anaesth 1998; 80: 525-7.

20. Beilin B, Yardeni IZ, Smolyarenko V, Zeidel A, Ram E, Mayburd E. Comparison of the Flexiblade levering laryngoscope with the English Macintosh laryngoscope in patients with a poor laryngoscopic view. Anaesthesia 2005; 60: 400-5.

21. Bein B, Worthmann F, Scholz J, Brinkmann F, Tonner PH, Steinfath $\mathrm{M}$, et al. A comparison of the intubating laryngeal mask airway and the Bonfils intubation fibrescope in patients with predicted difficult airways. Anaesthesia 2004; 59: 668-74.

22. Langeron O, Semjen F, Bourgain JL, Marsac A, Cros AM. Comparison of the intubating laryngeal mask airway with the fiberoptic intubation in anticipated difficult airway management. Anesthesiology 2001; 94: 968-72.

23. Lim TJ, Lim Y, Liu EH. Evaluation of ease of intubation with the GlideScope or Macintosh laryngoscope by anaesthetists in simulated easy and difficult laryngoscopy. Anaesthesia 2005; 60: 180-3.

24. Weiss M, Schwarz U, Gerber AC. Difficult airway management: comparison of the Bullard laryngoscope with the video-optical 
intubation stylet. Can J Anaesth 2000; 47: 280-4.

25. Hagberg CA, Westhofen P. A two-person technique for fiberscopeaided tracheal extubation/reintubation in intensive care unit (ICU) patients. J Clin Anesth 2003; 15: 467-70.
26. Rudolph C, Henn-Beilharz A, Gottschall R, Wallenborn J, Schaffranietz L. The unanticipated difficult intubation: rigid or flexible endoscope? Minerva Anestesiol 2007; 73: 567-74. 